\title{
Ablación de fibrilación auricular mediante energía de radiofrecuencia
}

\section{Radiofrequency ablation of atrial fibrillation}

\author{
Diego Andrés Rodríguez Guerrero ${ }^{a, b, c, *}$, Juan de Jesús Montenegro-Aldana ${ }^{a, b}$, \\ Luis Carlos Sáenz-Morales ${ }^{a, c}$ y Fermín García ${ }^{a, d}$
}

\author{
${ }^{\text {a }}$ Centro Internacional de Arritmias, Instituto de Cardiología, Fundación Cardioinfantil, Bogotá, Colombia \\ b Clínica Universitaria Colombia, Bogotá, Colombia \\ ' Facultad de Medicina, Universidad de La Sabana, Bogotá, Colombia \\ d Cardiac Electrophysiology, Division of Cardiology, Hospital of the University of Pennsylvania
}

Recibido el 3 de octubre de 2016; aceptado el 6 de octubre de 2016

Disponible en Internet el 5 de noviembre de 2016

\section{Introducción}

La base electrofisiológica de la fibrilación auricular requiere tanto un disparador que inicia la arritmia como un sustrato que pueda sostenerla ${ }^{1}$. Los desencadenantes más comunes de fibrilación auricular son latidos auriculares ectópicos que surgen de las extensiones musculares en las venas pulmonares $^{2}$. Estos factores desencadenantes pueden ser precipitados por la actividad intrínseca de plexos ganglionares cardíacos, agrupados en "clusters" en las uniones de las venas pulmonares con la aurícula izquierda ${ }^{3}$. Los factores existentes en la unión de las venas pulmonares con la aurícula izquierda asociados al crecimiento auricular que alberga fibrosis e inflamación, sirven como sustrato para sostener la arritmia. Con la persistencia de la fibrilación auricular, ocurre un cambio electrofisiológico adicional en el tejido auricular (acortamiento del período refractario auricular), que predispone al desarrollo de otros disparadores

\footnotetext{
* Autor para correspondencia.

Correo electrónico: diegoeef@gmail.com (D.A. Rodríguez Guerrero).
}

y circuitos que sostienen y perpetúan la arritmia. El mantenimiento del ritmo sinusal puede revertir estos cambios y mecanismos, de modo que la fibrilación auricular promueve fibrilación auricular y el ritmo sinusal promueve el ritmo sinusal ${ }^{4}$.

La ablación de la fibrilación auricular es una técnica terapéutica que utiliza energía de radiofrecuencia o congelación para destruir el tejido auricular que está implicado en la generación y el sostenimiento de la arritmia. La ablación por radiofrecuencia genera una corriente eléctrica alterna que atraviesa el tejido miocárdico atrial hasta el tejido más profundo. A temperaturas de $50^{\circ} \mathrm{C}$ o más, la mayoría de los tejidos se someten a necrosis de coagulación irreversible y luego evolucionan a un tejido miocárdico cicatricial no conductor $^{5}$. La crioablación destruye el tejido por congelación. El principal objetivo de la ablación de la fibrilación auricular es la desconexión eléctrica de los disparadores de las venas pulmonares del tejido auricular (a menudo llamado "aislamiento de las venas pulmonares") ${ }^{6}$. Para lograr este objetivo, la ablacioń se realiza alrededor de los orificios de las venas pulmonares aunque se pueden adicionar otras lesiones en los llamados electrogramas complejos fraccionados, vena cava superior, seno coronario, auriculilla 
izquierda, ablación en plexos ganglionares o diseñar líneas complementarias de ablación en casos de flutter auricular ${ }^{6}$.

El papel de la ablación con catéter en el tratamiento de la fibrilación auricular sigue evolucionando rápidamente, con mejoras en la eficacia y seguridad de los procedimientos. La eficacia de la ablación con catéter por radiofrecuencia para el mantenimiento del ritmo sinusal, es superior al tratamiento actual con medicamentos antiarrítmicos para el mantenimiento del ritmo sinusal en poblaciones de pacientes seleccionados. Se ha llevado a cabo una serie de consensos de la eficacia de la ablación con catéter de fibrilación auricular frente a la terapia con fármacos antiarrítmicos $^{7-12}$. La crioablación en el momento constituye una alternativa a la ablación punto a punto de la ablación por radiofrecuencia para alcanzar el aislamiento de las venas pulmonares $^{13}$. La evidencia que apoya la eficacia de la ablación con catéter es más fuerte para la fibrilación auricular paroxística en pacientes más jóvenes con poca o ninguna cardiopatía estructural ${ }^{14}$ y en los procedimientos realizados en centros de gran experiencia. De igual forma, los estudios han demostrado una reducción de los síntomas relacionados con la fibrilación auricular en estos contextos ${ }^{15}$. De otra parte, la evidencia es insuficiente para determinar si la ablación de fibrilación auricular con catéter reduce la mortalidad por todas las causas, los ataques cerebrovasculares y la insuficiencia cardiaca ${ }^{16}$. El ensayo clínico en curso (CABANA [Catheter Ablation Versus Antiarrhythmic Drug Therapy for Atrial Fibrillation] y el EAST [Early Therapy of Atrial Fibrillation for Stroke Prevention Trial]) deberían proporcionar nueva información para establecer si la ablación con catéter de la fibrilación auricular es superior a la terapia estándar con cualquiera de los fármacos de control del ritmo cardíaco en disminución de mortalidad total y otros puntos secundarios. Se pretende también evaluar si la aplicación temprana de una terapia de control del ritmo que implica ablación, antiarrítmicos, o ambos, puede afectar a los puntos finales de accidente cerebrovascular, muerte cardiovascular o insuficiencia cardiaca en comparación con el manejo médico habitual. Estos ensayos clínicos importantes ayudarán a establecer si la ablación con catéter proporciona un beneficio más allá de las mejoras en la calidad de vida ${ }^{16}$.

La decisión de someter un paciente a ablación de fibrilación auricular con catéter, depende de un gran número de variables, que incluyen tipo de fibrilación auricular (paroxística vs. persistente vs. largamente persistente), severidad de los síntomas, cardiopatía estructural, opciones alternativas, tales como control de la frecuencia o la terapia antiarrítmica mediante fármacos, probabilidad de complicaciones y preferencia del paciente. Es importante reconocer que la mayoría de los pacientes incluidos en los ensayos de ablación con catéter de fibrilación auricular han sido en general más jóvenes, sanos y con fibrilación auricular paroxística sintomática refractaria a uno o más medicamentos antiarrítmicos.

\section{Técnicas de ablación (aislamiento ostial, aislamiento antral, electrogramas complejos fraccionados, líneas adicionales, rotores)}

La ablación percutánea de la fibrilación auricular es un tratamiento eficaz ${ }^{17}$, en particular en los casos refractarios al manejo antiarrítmico. Considerando que los gatillos desencadenantes provienen de las venas pulmonares, la ablación implica la creación de lesiones circunferenciales en torno a las venas para aislarlas eléctricamente del resto de la aurícula izquierda ${ }^{18}$.

La ablación con catéter para fibrilación auricular persistente es más difícil y se asocia con resultados menos favorables ${ }^{19-22}$. Para mejorarlos, la ablación involucra el sustrato que mantiene la fibrilación auricular, mediante la creación de lesiones lineales en la aurícula izquierda ${ }^{23}$ y lesiones focales para eliminar señales auriculares fraccionadas ${ }^{24}$. En algunas oportunidades es necesaria la eliminación de gatillos extrapulmonares en auriculilla izquierda, seno coronario, venas cavas o ligamento de Marshall.

En el estudio STAR AF II (Approaches to Catheter Ablation for Persistent Atrial Fibrillation) que se trata de un ensayo aleatorizado con el objetivo de evaluar tres métodos diferentes para la ablación por radiofrecuencia en pacientes con fibrilación auricular persistente (aislamiento de venas pulmonares solamente, aislamiento de venas pulmonares más ablación de electrogramas complejos fraccionados o aislamiento de venas pulmonares más ablación lineal a través del techo de la aurícula izquierda y en el istmo mitral), no se encontró ninguna reducción en la tasa de la fibrilación auricular recurrente cuando se incorporaba ablación adicional a la necesaria para el aislamiento de venas pulmonares ${ }^{25}$.

Los intentos de mejorar los resultados de la ablación con catéter se han centrado en el sustrato que sostiene la fibrilación auricular después de que se instaura. Muchos estudios ahora desafían el concepto de que la fibrilación auricular se mantiene por ondas desordenadas autosostenibles localizadas (rotores) que mantienen la conducción fibrilatoria. Existen nuevas herramientas de mapeo que permiten la identificación y sirven de guía en la ablación de estos rotores con algunos resultados alentadores especialmente en pacientes con fibrilación auricular persistente o largamente persistente.

\section{Rol de la ablación en fibrilación auricular paroxística}

Dos estudios clínicos aleatorizados compararon la ablación con catéter por radiofrecuencia con la terapia con fármacos antiarrítmicos como tratamiento de control del ritmo de primera línea en pacientes con fibrilación auricular paroxística ${ }^{26}$. El estudio RAAFT (Radiofrequency Ablation Versus Antiarrhythmic Drugs for Atrial Fibrillation Treatment), comparó la eficacia de la ablación con catéter de fibrilación auricular con el de tratamiento con fármacos antiarrítmicos como opción de primera línea para el control del ritmo en 127 pacientes (88\% con fibrilación auricular paroxística) con mayor libertad de fibrilación auricular a un año (45\% frente a 28\%; $p=0,02$ ). El MANTRA-PAF (Medical Antiarrhythmic Treatment or Radiofrequency Ablation in Paroxysmal Atrial Fibrillation) $)^{27}$, comparó la ablación con catéter de la fibrilación auricular con la terapia mediante fármacos antiarrítmicos como terapia de primera línea en 294 pacientes. A los 24 meses de seguimiento, más pacientes en el grupo de ablación estaban libres de cualquier fibrilación auricular o fibrilación auricular sintomática, y la 
calidad de vida fue significativamente mejor. Sin embargo, la carga total de fibrilación auricular no fue significativamente diferente entre los dos grupos y las complicaciones mayores que requirieron intervención fueron más frecuentes en el grupo de ablación. Teniendo en cuentas estos estudios, se puede afirmar que la ablación de fibrilación auricular con radiofrecuencia puede ser considerada como terapia de primera línea en pacientes seleccionados, especialmente aquellos muy sintomáticos antes del manejo con fármacos antiarrítmicos cuando se desea una estrategia de control del ritmo ${ }^{28-33}$.

\section{Rol de la ablación en fibrilación auricular persistente y fibrilación auricular largamente persistente}

La seguridad y eficacia de la ablación con catéter está menos bien establecida para otras poblaciones de pacientes, especialmente aquellos con fibrilación auricular de larga persistencia, edad muy avanzada y con insuficiencia cardiaca significativa, incluyendo aquellos con miocardiopatía inducida por taquicardiaa ${ }^{34-41}$.

\section{Rol de la ablación en mayores de 75 años}

La ablación con catéter en pacientes de 75 años o más, se asocia con un resultado favorable a largo plazo en pacientes con fibrilación auricular paroxística, mientras que los resultados son menos prometedoras en persistente o de larga data. El perfil de seguridad de la ablación de fibrilación auricular en pacientes de 75 años o mayores, es comparable con la de pacientes más jóvenes ${ }^{42}$.

\section{Rol de la ablación en insuficiencia cardiaca}

Un número de ensayos clínicos pequeños han evaluado el papel de la ablación de fibrilación auricular con catéter en pacientes seleccionados con disfunción ventricular izquierda e insuficiencia cardiaca. Han demostrado una tasa razonable de éxito en el mantenimiento del ritmo sinusal con mejoría en la fracción de eyección del ventrículo izquierdo (FEVI) y síntomas ${ }^{43}$. El grado de mejoría de la FEVI varía según las características de cada paciente ${ }^{44,45}$. En los casos en que la disfunción del ventrículo izquierdo sea secundaria a la fibrilación auricular en sí, la ablación de ésta con catéter y el mantenimiento del ritmo sinusal, puede dar lugar a una mejoría notable. Así mismo, puede ser difícil determinar en esta población si los síntomas están relacionados con fibrilación auricular o falla cardiaca subyacente y si la fibrilación auricular ha contribuido a la disminución de la FEVI. El control óptimo de la frecuencia o la reversión a ritmo sinusal mediante cardioversión o con fármacos antiarrítmicos pueden ayudar a determinar la causalidad. Debido a la magnitud de la remodelación y a la enfermedad cardíaca subyacente, las tasas de recurrencia ${ }^{46}$ y de complicaciones son más altas en esta población. Un metaanálisis reportó que la eficacia del procedimiento sencillo de ablación de fibrilación auricular con catéter fue menor en los pacientes con disfunción sistólica, pero una tasa de éxito similar podría lograrse entre los pacientes con y sin disfunción sistólica con procedimientos repetidos. Tomados en su conjunto, la ablación por catéter puede ser razonable para el tratamiento de la fibrilación auricular sintomática en pacientes seleccionados con disfunción del ventrículo izquierdo significativa e insuficiencia cardiaca.

\section{Recomendaciones de la ablación de fibrilación auricular con catéter para mantener el ritmo sinusal ${ }^{47-49}$}

Estas directrices son el resultado de la revisión de las últimas guías propuestas por las Sociedades Americana (AHA/HRS), Europea (ESC/EHRA) y Canadiense de cardiología en la estrategia del control del ritmo mediante terapia de ablación. Tanto las guías europeas como las canadienses hacen énfasis en que los procedimientos de ablación de fibrilación auricular deben realizarse en centros de alta experiencia.

\section{Clase I}

1. La ablación de fibrilación auricular con catéter es útil para la fibrilación auricular paroxística sintomática refractaria o intolerante a por lo menos un antiarrítmico de clase I (propafenona, flecainida) o III (amiodarona, dronaderona, sotalol) y se desea una estrategia de control del ritmo (nivel de evidencia A).

2. Antes de considerar la ablación de fibrilación auricular con catéter, se recomienda que en cada paciente se evalúen los riesgos y resultados de la intervención (nivel de evidencia C).

\section{Clase Ila}

1. La ablación de fibrilación auricular con catéter es razonable para algunos pacientes con fibrilación auricular sintomática persistente, refractaria o intolerante a por lo menos un antiarrítmico de clase I (propafenona, flecainida) o III (amiodarona, sotalol) (nivel de evidencia A).

2. En los pacientes con fibrilación auricular paroxística sintomática recurrente, la ablación con catéter es una estrategia de control del ritmo inicial razonable antes de los ensayos terapéuticos con fármacos antiarrítmicos, después de sopesar los riesgos y los resultados de la terapia farmacológica comparada con la terapia de ablación ${ }^{40,42}$ (nivel de evidencia $\mathrm{B}$ ).

3. Cuando se planee la ablación de fibrilación auricular con catéter se debe considerar continuar la terapia de anticoagulación oral con antagonistas de la vitamina $\mathrm{K}$ durante el procedimiento de ablación, buscando mantener un INR cercano a 2 (nivel de evidencia B)

\section{Clase IIb}

1. La ablación con catéter de fibrilación auricular puede ser considerada para pacientes sintomáticos con fibrilación auricular largamente persistentes (> 12 meses) refractaria o intolerante a por lo menos un medicamento antiarrítmico clase I o III cuando se desea la estrategia de control del ritmo (nivel de evidencia $B$ ). 
2. La ablación de fibrilación auricular con catéter se puede considerar antes de iniciar el tratamiento con medicamentos antiarrítmicos de clase I o III para pacientes con fibrilación auricular persistente sintomática cuando se desea una estrategia de control del ritmo (nivel de evidencia C).

\section{Clase III: Daño}

1. La ablación de fibrilación auricular con catéter no debe realizarse en pacientes que no pueden ser tratados con la terapia anticoagulante durante y después del procedimiento (nivel de evidencia $C$ ).

2. La ablación de fibrilación auricular con catéter para restaurar el ritmo sinusal no se debe realizar con la única intención de obviar la necesidad de anticoagulación. (nivel de evidencia C).

\section{Anticoagulación periprocedimiento de ablación de fibrilación auricular}

Debido al riesgo bien establecido de ictus o ataque isquémico transitorio periprocedimiento asociado con la ablación de fibrilación auricular con catéter, hay consenso en que se indica la anticoagulación para prevenir la tromboembolia durante el procedimiento sin importar el riesgo tromboembólico de base del paciente. Las recomendaciones detalladas de consenso sobre el enfoque de la anticoagulación antes, durante y después de ablación con catéter ya han sido publicadas en capítulos previos. Tanto la heparina intra-procedimiento y la anticoagulación oral se recomiendan por dos meses o más después del procedimiento. La ablación de fibrilación auricular con catéter no debe realizarse en pacientes que no pueden ser tratados con la terapia anticoagulante durante y después del procedimiento. Varios informes indican que la ablación con catéter de fibrilación auricular puede llevarse a cabo con menos complicaciones cuando la anticoagulación con warfarina oral no fue suspendida periprocedimiento. Varios centros han informado su experiencia con el uso de inhibidores directos de la trombina $y$ del factor Xa.

\section{Recurrencias después de la ablación con catéter}

Las recurrencias de fibrilación auricular tras la ablación con catéter son comunes durante los primeros tres meses y no predicen el éxito a largo plazo, a pesar de que están asociados con mayor riesgo de reconexión de venas pulmonares y re-hospitalización. Por tanto, cuando se presenta fibrilación auricular poco después de la ablación con catéter, se debe considerar el manejo farmacológico del control del ritmo en lugar de repetir la ablación de manera temprana. Los pacientes que desarrollan fibrilación auricular persistente dentro de los tres primeros meses después de la ablación, pueden requerir cardioversión. La fibrilación auricular recurrente después de tres meses suele ser una indicación de la reconexión de las venas pulmonares y puede ser manejada repitiendo el procedimiento o con el inicio de antiarrítmicos. Varios centros han informado recurrencias más allá de un año después de la ablación.

\section{Complicaciones de la ablación de fibrilación auricular}

La ablación con catéter de fibrilación auricular se asocia a riesgos importantes de complicaciones mayores. Una encuesta internacional de los procedimientos de ablación con catéter por radiofrecuencia en el año 2010, encontró una incidencia de $4,5 \%$ de complicaciones mayores, incluyendo una tasa de 1,3\% de taponamiento cardíaco, una tasa de $0,94 \%$ de ictus o ataque isquémico transitorio, una tasa de $0,04 \%$ de fístula atrio-esofágica y un 0,15\% de mortalidad. Un registro observacional multinacional europeo reportó una tasa de complicaciones del $7,7 \%$, de los cuales $1,7 \%$ eran complicaciones mayores. Muchos de los datos sobre las tasas de complicaciones se derivan de centros experimentados o registros voluntarios.

\section{Conflicto de intereses}

Los autores declaran no tener ningún conflicto de intereses.

\section{Bibliografía}

1. Moe GK. Evidence for reentry as a mechanism of cardiac arrhythmias. Rev Physiol Biochem Pharmacol. 1975;72:55-81.

2. Cheung DW. Electrical activity of the pulmonary vein and its interaction with the right atrium in the guinea-pig. J Physiol. 1981;314:445-56

3. Lin J, Scherlag BJ, Zhou J, et al. Autonomic mechanism to explain complex fractionated atrial electrograms (CFAE). J Cardiovasc Electrophysiol. 2007;18:1197-205.

4. Wijffels MC, Kirchhof CJ, Dorland R, Allessie MA. Atrial fibrillation begets atrial fibrillation: a study in awake chronically instrumented goats. Circulation. 1995;92:1954-68.

5. Haines DE. The biophysics of radiofrequency catheter ablation in the heart: the importance of temperature monitoring. Pacing Clin Electrophysiol. 1993;16:586-91.

6. Haissaguerre M, Jais P, Shah DC, Takahashi A, Hocini M, Quiniou $\mathrm{G}$, et al. Spontaneous initiation of atrial fibrillation by ectopic beats originating in the pulmonary veins. $\mathrm{N}$ Engl $\mathrm{J}$ Med. 1998;339:659-66.

7. Calkins H, Reynolds MR, Spector P, et al. Treatment of atrial fibrillation with antiarrhythmic drugs or radiofrequency ablation: two systematic literature reviews and meta-analyses. Circ Arrhythm Electrophysiol. 2009;2:349-61.

8. Bonanno C, Paccanaro M, La Vecchia L, et al. Efficacy and safety of catheter ablation versus antiarrhythmic drugs for atrial fibrillation: a meta-analysis of randomized trials. J Cardiovasc Med (Hagerstown). 2010;11:408-18.

9. Nair GM, Nery PB, Diwakaramenon S, et al. A systematic review of randomized trials comparing radiofrequency ablation with antiarrhythmic medications in patients with atrial fibrillation. J Cardiovasc Electrophysiol. 2009;20:138-44.

10. Parkash R, Tang AS, Sapp JL, Wells G. Approach to the catheter ablation technique of paroxysmal and persistent atrial fibrillation: a meta-analysis of the randomized controlled trials. J Cardiovasc Electrophysiol. 2011;22:729-38.

11. Piccini JP, Lopes RD, Kong $M$ H, et al. Pulmonary vein isolation for the maintenance of sinus rhythm in patients with atrial fibrillation: a meta-analysis of randomized, controlled trials. Circ Arrhythm Electrophysiol. 2009;2:626-33. 
12. Terasawa T, Balk EM, Chung M, et al. Systematic review: comparative effectiveness of radiofrequency catheter ablation for atrial fibrillation. Ann Intern Med. 2009;151:191-202.

13. Packer DL, Kowal RC, Wheelan KR, et al. balloon ablation of pulmonary veins for paroxysmal atrial fibrillation: first results of the North American Arctic Front (STOP AF) Pivotal Trial. J Am Coll Cardiol. 2013;61:1713-23.

14. Leong-Sit P, Zado E, Callans DJ, et al. Efficacy and risk of atrial fibrillation ablation before 45 years of age. Circ Arrhythm Electrophysiol. 2010;3:452-7.

15. Al-Khatib SM, Allen Lapointe N, Chatterjee R, et al. Treatment of Atrial Fibrillation. Comparative Effectiveness Review 119. (Prepared by the Duke Evidence-based Practice Center under Contract No. 290-2007-10066-I.) AHRQ Publication No.13-EHC095-EF. Rockville, MD: Agency for Healthcare Research and Quality; [June 2013]. Disponible en: http://www.effectivehealthcare.ahrq.gov/ehc/products/358/ 1559/atrial-fibrillation-report-130628.pdf

16. Verma A, Macle L, Cox J, Skanes AC. Canadian Cardiovascular Society atrial fibrillation guidelines 2010: catheter ablation for atrial fibrillation/atrial flutter. Can J Cardiol. 2011;27:60-6.

17. Wilber DJ, Pappone C, Neuzil P, et al. Comparison of antiarrhythmic drug therapy and radiofrequency catheter ablation in patients with paroxysmal atrial fibrillation: a randomized controlled trial. JAMA. 2010;303:333-40.

18. Stabile G, Bertaglia E, Senatore G, et al. Catheter ablation treatment in patients with drug-refractory atrial fibrillation: a prospective, multi-centre, randomized, controlled study (Catheter Ablation For The Cure Of Atrial Fibrillation Study). Eur Heart J. 2006;27:216-21.

19. Parkash R, Verma A, Tang AS. Persistent atrial fibrillation: current approach and controversies. Curr Opin Cardiol. 2010;25:1-7.

20. Brooks AG, Stiles MK, Laborderie J, et al. Outcomes of longstanding persistent atrial fibrillation ablation: a systematic review. Heart Rhythm. 2010;7:835-46.

21. Verma A, Mantovan R, Macle L, et al. Substrate and Trigger Ablation for Reduction of Atrial Fibrillation (STAR AF): a randomized, multicentre, international trial. Eur Heart J. 2010;31:1344-56.

22. Fassini G, Riva S, Chiodelli R, et al. Left mitral isthmus ablation associated with PV isolation: long-term results of a prospective randomized study. J Cardiovasc Electrophysiol. 2005;16:1150-6.

23. Jaïs $P$, Hocini M, Hsu LF, et al. Technique and results of linear ablation at the mitral isthmus. Circulation. 2004;110:2996-3002.

24. Nademanee K, McKenzie J, Kosar E, et al. A new approach for catheter ablation of atrial fibrillation: mapping of the electrophysiologic substrate. J Am Coll Cardiol. 2004;43:2044-53.

25. Verma A, Jiang CY, Betts TR, et al. Approaches to catheter ablation for persistent atrial fibrillation. N Engl J Med. 2015;372:1812-22.

26. Morillo C, Verma A, Connolly SJ, et al. Radiofrequency ablation vs. antiarrhythmic drugs as first-line treatment of paroxysmal atrial fibrillation (RAAFT 2): a randomized trial. JAMA. 2014;311:692-700.

27. Jons C, Hansen PS, Johannessen A, et al. The Medical ANtiarrhythmic Treatment or Radiofrequency Ablation in Paroxysmal Atrial Fibrillation (MANTRA-PAF) trial: clinical rationale, study design, and implementation. Europace. 2009;11:917-23.

28. Jais P, Cauchemez B, Macle L, et al. Catheter ablation versus antiarrhythmic drugs for atrial fibrillation: the A4 study. Circulation. 2008;118:2498-505.

29. Oral H, Pappone C, Chugh A, et al. Circumferential pulmonaryvein ablation for chronic atrial fibrillation. N Engl J Med. 2006;354:934-41.

30. Mont L, Bisbal F, Hernandez-Madrid A, et al. Catheter ablation vs. antiarrhythmic drug treatment of persistent atrial fibrillation: a multicentre, randomized, controlled trial (SARA study). Eur Heart J. 2014;35:501-7.

31. Cosedis NJ, Johannessen A, Raatikainen P, et al. Radiofrequency ablation as initial therapy in paroxysmal atrial fibrillation. $\mathrm{N}$ Engl J Med. 2012;367:1587-95.

32. Haissaguerre M, Hocini M, Sanders P, et al. Catheter ablation of long-lasting persistent atrial fibrillation: clinical outcome and mechanisms of subsequent arrhythmias. J Cardiovasc Electrophysiol. 2005;16:1138-47.

33. Di Biase L, Burkhardt JD, Mohanty P, et al. Periprocedural stroke and management of major bleeding complications in patients undergoing catheter ablation of atrial fibrillation: the impact of periprocedural therapeutic international normalized ratio. Circulation. 2010;121:2550-6.

34. Kwak JJ, Pak HN, Jang JK, et al. Safety and convenience of continuous warfarin strategy during the periprocedural period in patients who underwent catheter ablation of atrial fibrillation. J Cardiovasc Electrophysiol. 2010;21:620-5.

35. Gaztanaga L, Frankel DS, Kohari M, et al. Time to recurrence of atrial fibrillation influences outcome following catheter ablation. Heart Rhythm. 2013;10:2-9.

36. Medi C, Sparks PB, Morton JB, et al. Pulmonary vein antral isolation for paroxysmal atrial fibrillation: results from long-term follow-up. J Cardiovasc Electrophysiol. 2011;22:137-41.

37. Ouyang F, Tilz R, Chun J, et al. Long-term results of catheter ablation in paroxysmal atrial fibrillation: lessons from a 5-year follow-up. Circulation. 2010;122:2368-77.

38. Sawhney N, Anousheh R, Chen WC, et al. Five-year outcomes after segmental pulmonary vein isolation for paroxysmal atrial fibrillation. Am J Cardiol. 2009;104:366-72.

39. Wokhlu A, Hodge DO, Monahan KH, et al. Long-term outcome of atrial fibrillation ablation: impact and predictors of very late recurrence. J Cardiovasc Electrophysiol. 2010;21:1071-8.

40. Cappato R, Calkins H, Chen SA, et al. Updated worldwide survey on the methods, efficacy, and safety of catheter ablation for human atrial fibrillation. Circ Arrhythm Electrophysiol. 2010;3:32-8.

41. Arbelo E, Brugada J, Hindricks G, et al. ESC-EURObservational Research Programme: the Atrial Fibrillation Ablation Pilot Study, conducted by the European Heart Rhythm Association. Europace. 2012;14:1094-103.

42. Metzner I, Wissner E, Tilz RR, et al. Ablation of atrial fibrillation in patients $\geq 75$ years: long-term clinical outcome and safety. DOI: http://dx.doi.org/10.1093/europace/euv229 euv229 First published online: 29 January 2016.

43. Khan MN, Jais $P$, Cummings $J$, et al. Pulmonary-vein isolation for atrial fibrillation in patients with heart failure. N Engl $\mathrm{J}$ Med. 2008;359:1778-85.

44. Jones DG, Haldar SK, Hussain W, et al. A randomized trial to assess catheter ablation versus rate control in the management of persistent atrial fibrillation in heart failure. J Am Coll Cardiol. 2013;61:1894-903.

45. Cha YM, Wokhlu A, Asirvatham SJ, et al. Success of ablation for atrial fibrillation in isolated left ventricular diastolic dysfunction: a comparison to systolic dysfunction and normal ventricular function. Circ Arrhythm Electrophysiol. 2011;4:724-32.

46. Wilton SB, Fundytus A, Ghali WA, et al. Meta-analysis of the effectiveness and safety of catheter ablation of atrial fibrillation in patients with versus without left ventricular systolic dysfunction. Am J Cardiol. 2010;106:1284-91.

47. Calkins H, Brugada J, Packer DL, et al. HRS/EHRA/ECAS expert consensus statement on catheter and surgical ablation of atrial fibrillation: recommendations for personnel, policy, procedures and follow-up: a report of the Heart Rhythm Society (HRS) Task Force on Catheter and Surgical Ablation of Atrial Fibrillation developed in partnership with the European Heart Rhythm Association (EHRA) and the European Cardiac Arrhythmia Society 
(ECAS); in collaboration with the American College of Cardiology (ACC), American Heart Association (AHA), and the Society of Thoracic Surgeons (STS): endorsed and approved by the governing bodies of the American College of Cardiology, the American Heart Association, the European Cardiac Arrhythmia Society, the European Heart Rhythm Association, the Society of Thoracic Surgeons, and the Heart Rhythm Society. Europace. 2007;9:335-79.

48. Calkins H, Kuck KH, Cappato R, et al. 2012 HRS/EHRA/ECAS expert consensus statement on catheter and surgical ablation of atrial fibrillation: recommendations for patient selection, procedural techniques, patient management and follow-up, definitions, endpoints, and research trial design: a report of the Heart Rhythm Society (HRS) Task Force on Catheter and
Surgical Ablation of Atrial Fibrillation. Developed in partnership with the European Heart Rhythm Association (EHRA), a registered branch of the European Society of Cardiology (ESC) and the European Cardiac Arrhythmia Society (ECAS); and in collaboration with the American College of Cardiology (ACC), American Heart Association (AHA), the Asia Pacific Heart Rhythm Society (APHRS), and the Society of Thoracic Surgeons (STS). Heart Rhythm. 2012;9:632-96.

49. January CT, Wann LS, Alpert JS, et al. 2014 AHA/ACC/HRS guideline for the management of patients with atrial fibrillation: executive summary: a report of the American College of Cardiology/American Heart Association Task Force on practice guidelines and the Heart Rhythm Society. Circulation. 2014;130:2071-104. 